\title{
Implants that vanish
}

\author{
Soft biomaterials for implantation in the body are increasingly designed to be functional for a finite time and to then \\ disappear via degradation or resorption.
}

M any implanted biomaterials have to be removed from the body when they fail or are no longer needed, often via surgery. Yet, the permanence of an implant is not always essential, especially when the implant's function is only needed transiently. This is the case of implants that are meant to, for example, sense signals from tissue (such as bioresorbable silicon electronics for implantation in the brain; Nature 530, 71-76; 2016) or induce the regeneration of damaged tissue (such as clinically available self-degrading coronary stents; Nat. Rev. Cardiol. 13, 719-729; 2016). Also, transient implants may evade any longterm biocompatibility issues.

Transient implants typically involve soft or thin biomaterials and structures that enzymatically degrade or slowly dissolve, or that are resorbed by surrounding tissue (via bulk or surface erosion, and as a result of cellular activity) after an approximately predefined period of stable function, such as the monitoring of recovery from traumatic injury or of responses to treatment. A recent example of such implants, reported by John Rogers and colleagues, is a bioresorbable pressure sensor, made of ultrathin silicon and silica structures, that can monitor intracranial pressure in awake rats for more than three weeks before dissolving. Now, Rogers and colleagues report, in an Article in this issue, bioresorbable spectroscopic probes (pictured) that can monitor cerebral temperature, oxygenation and neural activity in the brains of freely moving mice. The implanted probes, which consist of multiwavelength photodetectors, multilayer filters and fibre-optic probes made of dissolvable, thin, Si-based materials and polymer (poly(lactic-co-glycolic acid)), do not cause any measureable immune responses, gradually dissolve, and have completely disappeared seven weeks after implantation, at which point the concentration of the dissolved elements in the blood and tissues of the mice have returned to normal levels.

In another Article in this issue, Xinyi $\mathrm{Su}$, Xian Jun Loh, Gopal Lingam and colleagues describe a bioresorbable thermogelling co-polymer that functions as a tamponade during vitreoretinal surgery for the treatment of retinal detachment. Approximately three months after being injected into the eyes of rabbits and

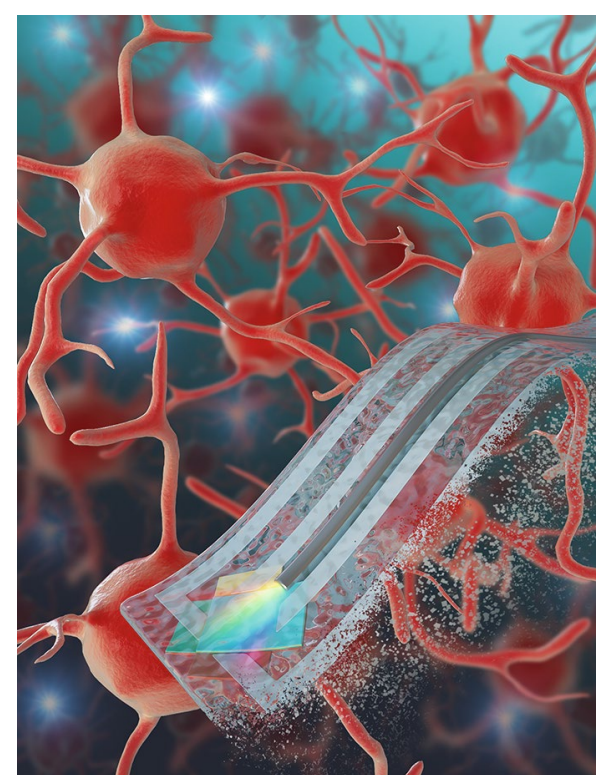

Credit: W. Bai and J. Rogers, Northwestern University

macaques following vitrectomy (the surgical removal of the vitreous), most of the gelled polymer had degraded and been substituted by a vitreous body with properties similar to the native vitreous. Unlike silicone oil - the clinical gold standard for a longterm tamponade - the transparency and refractive index of the polymer gel are similar to the native vitreous and thus would allow the patient to retain their vision during post-operative recovery.

There are applications that demand biodegradable implants that stay adhered to tissue. This is the case of barriers for the reduction of the formation of post-operative pericardial adhesions - scar tissue that forms between the heart and the sternum, as a result of the healing process occurring after cardiac surgery. Existing commercial adhesion barriers degrade too quickly or do not stay in place. As shown in an Article by Joseph Woo, Eric Appel and colleagues included in this issue, a slowly degradable crosslinked hydrogel made of a dodecylmodified cellulose derivative and polymer nanoparticles can be sprayed onto the heart during cardiac surgery, is retained on the pericardium of rats for two weeks, and reduced the incidence and severity of cardiac adhesions in sheep that had undergone surgery for cardiopulmonary bypass. The viscoelastic supramolecular hydrogel is biocompatible, simple to produce at scale and easy to handle, and therefore might become a more effective adhesion barrier for deployment during abdominal surgeries.

For implanted soft biomaterials, making them viscoelastic is essential when they need to be attached to moving organs with intrinsic dynamic deformations, such as the heart. Also in an Article in this issue, Huajian Gao, Ning Sun, Lei Wang and colleagues demonstrate that an epicardial patch made of an ionically crosslinked starch hydrogel, and whose properties were optimized computationally (via finiteelement modelling) for the treatment of myocardial infarction, reliably restrained ventricular dilation and prevented left ventricular remodelling and cardiac failure in rats. The optimal performance of the patch resulted from it being near the 'gel point' (when the viscosity of a fluid changes abruptly as it gels), where fluidity and viscous dissipation are balanced. As noted by Milica Radisic and colleagues in an accompanying News \& Views, "a version of the optimal patch that could be placed on the heart endoscopically or via transthoracic injection would be desirable, to reduce the risks and complications associated with open-heart surgery".

When discussing the removability of non-degradable high-density electrode arrays for recording activity in the brain, Laura Cabrera and colleagues contemplate, in a Comment included in this issue, "what if deleterious side effects or improper placement is observed, and the device cannot be removed? What if removal of the device is a greater risk to morbidity and/or mortality than simply leaving the foreign body in the brain?". In future, such high-density arrays of electrodes could be mechanically compliant with brain tissue, cause minimal immune responses and, after their operational lifetime, vanish.

Published online: 7 August 2019 https://doi.org/10.1038/s41551-019-0449-5 